\title{
2 EdUCAÇÃo PERMANENTE: PRÁtICAS E PROCESSOS DA ENFERMAGEM EM SAÚDE MENTAL ${ }^{1}$
}

\author{
| Amanda dos Santos Mota ${ }^{2}$; Ana Lúcia Abrahão da Silva ${ }^{3}$ Ândrea Cardoso de Souza $\mid$
}

\section{RESUMO}

CONTEXTO: Com a Reforma Psiquiátrica brasileira, o processo de trabalho da equipe de enfermagem se deparou com novas convocações no sentido de valorizar o relacionamento terapêutico, aumentando a capacidade de escuta e de diálogo com os usuários.

OBJETIVO: Analisar o processo de Educação Permanente (EP) em um hospital psiquiátrico, a partir da dinâmica do trabalho da equipe de enfermagem.

METODOLOGIA: Pesquisa descritiva, qualitativa, do tipo intervenção, em que foram empregadas a observação, diário de campo e acompanhamento do grupo. Participaram deste estudo 27 pessoas, sendo a maioria enfermeiros e técnicos de enfermagem que frequentavam o grupo de EP, entre o período de outubro de 2012 a abril de 2013, totalizando oito encontros. Os critérios de inclusão dos participantes foram: possuir vínculo empregatício com o hospital; atuar na profissão de enfermagem há mais de um ano; frequentar regularmente o espaço do grupo de discussão. Os critérios de exclusão foram: profissionais sem experiência no campo da saúde mental e profissionais externos ao hospital. Os dados foram submetidos à análise de conteúdo temática. Esta pesquisa foi aprovada pelo Comitê de Ética e Pesquisa do Hospital Universitário Antônio Pedro, da Universidade Federal Fluminense, tendo sido aprovado sob n ${ }^{\circ} 125.286$.

RESULTADOS: Após a análise do material foram elencadas as seguintes categorias: (i) Ser ouvido; (ii) A dinâmica processual do trabalho em saúde.

CONCLUSÕES: Há dificuldades para a equipe de enfermagem se fazer presente no espaço de EP, tanto pela própria resistência em se permitir ocupar outros lugares, quanto pelos demais membros da equipe.

PALAVRAS-CHAVE: Educação continuada; Enfermagem psiquiátrica; Ambiente de trabalho

\section{RESUMEN}

"Ongoing education: Practices and processes related to mental health nursing"

BACKGROUND: New summons were presented to the work process of the nursing team by the Brazilian Psychiatric Reform aiming at the enhancement of the therapeutic relationship, increasing listening skills and dialogue with users.

AIM: To analyze the process of Permanent Education (PE) in a psychiatric hospital, from the dynamics of the nursing team work.

METHODS: This is a descriptive and qualitative research, of the intervention type, in which observation, field diary, and group monitoring were employed. The study included 27 people, mostly nurses and nursing technicians who attended the PE group, in the period from October 2012 to April 2013, totalling eight meetings. The inclusion criteria of the participants were: to be legally employed in the hospital; work in the nursing profession for more than one year; regularly attend the space of the discussion group. Exclusion criteria were: professionals with no experience in the field of mental health and external professionals to the hospital. The data were submitted to thematic content analysis. This study was approved by the Research Ethics Committee of the University Hospital Antônio Pedro, Fluminense Federal University, and was approved under No. 125,286.

RESULTS: After analyzing the material were listed the following categories: (i) being heard; (ii) The dynamic process of health work. CONCLUSIONS: There are difficulties for the nursing staff to be present within the space of $\mathrm{PE}$; both by the resistance to allow the occupation of other places, as for the other team members.

\section{DESCRIPTORES: Educación permanente; Enfermería} psiquiátrica; Ambiente de trabajo

\section{ABSTRACT \\ "Educación permanente: Prácticas y procesos en enfermería de la salud mental"}

BACKGROUND: New summons were presented to the work process of the nursing team by the Brazilian Psychiatric Reform aiming at the enhancement of the therapeutic relationship, increasing listening skills and dialogue with users.

AIM: To analyze the process of Permanent Education (PE) in a psychiatric hospital, from the dynamics of the nursing team work.

METHODS: This is a descriptive and qualitative research, of the intervention type, in which observation, field diary, and group monitoring were employed. The study included 27 people, mostly nurses and nursing technicians who attended the PE group, in the period from October 2012 to April 2013, totalling eight meetings. The inclusion criteria of the participants were: to be legally employed in the hospital; work in the nursing profession for more than one year; regularly attend the space of the discussion group. Exclusion criteria were: professionals with no experience in the field of mental health and external professionals to the hospital. The data were submitted to thematic content analysis. This study was approved by the Research Ethics Committee of the University Hospital Antônio Pedro, Fluminense Federal University, and was approved under No. 125,286.

RESULTS: After analyzing the material were listed the following categories: (i) being heard; (ii) The dynamic process of health work. CONCLUSIONS: There are difficulties for the nursing staff to be present within the space of $\mathrm{PE}$; both by the resistance to allow the occupation of other places, as for the other team members.

\section{KEYWORDS: Continuing education; Psychiatric nursing; Working environment}

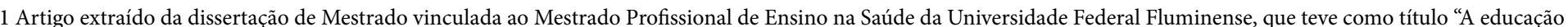
permanente no cotidiano das novas práticas em saúde mental".

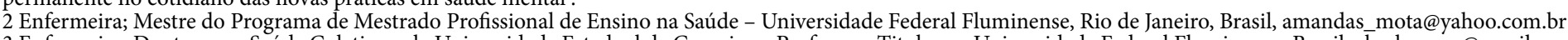

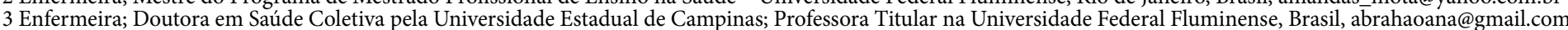

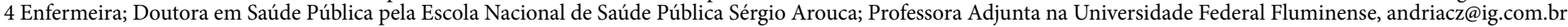

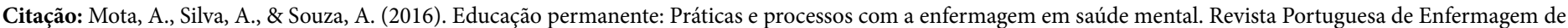
Saúde Mental (Spe. 4), 09-16.

Submetido em 20-12-2015 / Aceite em 30-05-2016 


\section{INTRODUÇÃO}

No conjunto das políticas de saúde desenhadas no cenário brasileiro, a partir da $8^{\text {a }}$ Conferência Nacional de Saúde (1986), a Reforma Psiquiátrica Brasileira (RPB) é um marco. Ela vêm possibilitando a incorporação de novos conhecimentos e a implementação de novas propostas de assistência ao usuário com sofrimento psíquico, no sentido de romper com as práticas asilares da organização hospitalocêntrica, dando lugar as práticas que resgatem a autonomia, autoconhecimento, e aumento da capacidade de fazer escolhas (Rocha, 2012). Tal reformulação da assistência exigiu uma mudança na prática do cuidado psiquiátrico e da relação terapêutica entre usuário-profissional e usuário-instituição (Borba, Guimarães, Mazza, e Maftum, 2012). A reforma, no campo da psiquiatria, implicou repensar o modelo de assistência tradicional, por meio da vinculação da saúde mental aos conceitos de cidadania e qualidade de vida, a fim de que fosse possível a inclusão social da pessoa com transtorno mental promovendo respeito às diferenças e singularidades dela.

A enfermagem, como uma profissão que compõem a equipe de saúde, nesse novo cenário, é convidada a ir além das ações de conter, vigiar e medicar, que durante tantos anos resumiram a sua participação no processo de cuidado em saúde mental. Nessa configuração, o processo de trabalho, principalmente da equipe de enfermagem, se deparou com novas convocações na assistência, no sentido de valorizar o relacionamento terapêutico, aumentando a capacidade de escuta e de diálogo com os usuários.

O compartilhar experiências e saberes no trabalho cooperativo pode ser um elemento de contribuição para que a equipe de enfermagem possa reconhecer a necessidade de se aproximar da proposta de substituição do modelo de assistência no campo psiquiátrico e repensar a prática para além de um saber eminentemente técnico e disciplinador. Assim, sentimentos, atitudes, cooperação, solidariedade e responsabilidade social estarão envolvidos num mesmo propósito, colocados como elementos necessários ao processo de aprendizagem.

Entretanto, a inovação no campo das ideias e estruturação na saúde mental não foi acompanhada, na mesma proporção, de inovações no campo da formação em saúde e trabalho em saúde na qual, segundo Merhy e Abrahão (2014), um produz o outro. E é nessa perspectiva que os profissionais devem ser vistos como um componente dinâmico que necessita de apoio e investimento permanente (Santos, Portugal, Silva, Souza e Abrahão, 2015), no sentido de acompanhar as mudanças desta nova política que instaura uma outra prática de cuidado.
Uma prática marcada por conflitos derivados da transição paradigmática nos modos de intervenção em saúde mental, em que a prática disciplinadora e técnica é confrontada com uma prática cidadã e produtora de sujeitos. E é nessa configuração que a Educação Permanente em Saúde (EPS) apresenta-se como eixo possibilitador de reflexão e mudanças destas práticas no campo da saúde e, no nosso caso, da Saúde Mental.

A educação permanente (EP) é compreendida como sendo um processo educativo contínuo, de revitalização e superação pessoal e profissional, de modo individual e coletivo, com objetivo de qualificação, reafirmação ou reformulação de valores, construindo relações integradoras entre os sujeitos envolvidos para uma praxe crítica e criadora (Slomp Junior, Feuerwerker, \& Land, 2015). Se propõe, a partir da análise coletiva, problematizar o processo de trabalho, na perspectiva que seus atores possam ser capazes de se responsabilizarem mutuamente pela produção de autonomia e de cuidados em busca da integralidade da assistência (Ceccim, 2012).

Compreende-se, neste artigo, que a educação deve ser construída pelo sujeito na sua relação com os outros e com o mundo. Ela pode ser considerada instrumento de transformação social quando forma profissionais de saúde para serem agentes de mudança, críticos, criativos, reflexivos, propositivos, capazes de assumir, no conjunto das lutas sociais, sua condição de agente ativo de transformação da sociedade e de si próprio, na conquista de direitos e justiça social (Slomp Junior et al., 2015). Baseada na aprendizagem significativa, a EPS também propõe que essa análise seja desenvolvida na interlocução, em rodas de conversas sobre os problemas e dificuldades vivenciados no cotidiano da produção do cuidado, da gestão, da formação dos trabalhadores. Diante desse contexto, este artigo apresenta uma parte da pesquisa A educação permanente no cotidiano das novas práticas em saúde mental, que objetivou analisar o processo de EPS num hospital psiquiátrico público no estado do Rio de Janeiro, a partir da dinâmica de participação da equipe de enfermagem em grupos de discussão sobre o trabalho em saúde mental, entre os anos de 2012 e 2013.

Estudar modos de contribuir com a melhora da formação dos profissionais e da assistência prestada aos usuários com transtornos mentais é o foco deste artigo, com base no trabalho da EP como um caminho de debate sobre o processo de trabalho e abertura de novas possibilidades no cuidado. 


\section{METODOLOGIA}

O estudo toma como desenho uma pesquisa qualitativa, descritiva, do tipo intervenção, realizada num hospital psiquiátrico público no estado do Rio de Janeiro. Nesse tipo de pesquisa tem-se as práticas sociais e os referenciais que lhe dão sentido se produzindo simultaneamente. $\mathrm{O}$ conhecimento e a ação sobre a realidade são desenvolvidos no curso da investigação, de acordo com a análise e decisões da coletividade envolvida. Ou seja, a presença ativa dos participantes do estudo é garantida no caminhar do processo.

Utilizaram-se como técnicas de coleta de dados a observação não participante, o diário de campo e os grupos de discussão sobre o trabalho em saúde mental, no período de outubro de 2012 a abril de 2013, totalizando oito encontros. O grupo estudado foi instituído há quatro anos no hospital psiquiátrico, que fez parte do estudo, com objetivo de viabilizar a discussão de casos relacionados aos pacientes entre os profissionais que compõem a equipe multiprofissional do hospital.

Em busca de enriquecer o movimento de discussão no grupo, foi elaborado um caso analisador a partir da criação de um caso fictício, encenado a partir de pequenas esquetes por graduandas de enfermagem, representando a equipe de enfermagem (enfermeiros e técnicos de enfermagem).

O caso analisador, ora denominado de 'analisador artificial' pela Análise Institucional (AI), é construído pelo analista para ser utilizado como provocador das discussões em grupo. No caso deste estudo, ele foi estabelecido com base em situações colhidas a partir da produção do diário de campo e da observação, e que conduziram a construção das esquetes, do caso analisador, empregadas no grupo estudado. O grupo foi 'exposto' ao caso por meio de esquetes teatrais realizada no dia 24 de abril de 2013, com duração de aproximadamente uma hora, no qual se deu a representação e discussão a partir de elementos apresentados: o silêncio dos profissionais nas reuniões; o sono; a resistência em sentar na roda; a presença de figuras de poder da instituição durante as reuniões; trocas de saberes a partir das experiências e o desejo de fazer diferente.

Participaram do estudo 27 profissionais de enfermagem, elegíveis após aplicação dos seguintes critérios de inclusão: possuir vínculo empregatício com o hospital; atuar na profissão de enfermagem há mais de um ano; frequentar regularmente o espaço do grupo de discussão. Os critérios de exclusão considerados foram: profissionais sem experiência no campo da saúde mental; profissionais externos ao hospital.
O espaço frequentado de maneira mais assídua foi o grupo de EP que ocorre às quartas-feiras onde acontecia apresentação de casos, e que está constituída há aproximadamente quatro anos. A escolha desse espaço se justifica pelo interesse no movimento da equipe de enfermagem (composta pelo enfermeiro, técnico de enfermagem e auxiliar de enfermagem) participar ou ter um espaço para debate acerca de seu processo de trabalho. E também por esse espaço, apesar de aberto a todos do hospital, ser capaz de reunir uma representatividade desses profissionais (27 em média) oriundos das diversas enfermarias/setores do hospital.

A observação de campo gerou a produção de um diário, no qual foram descritas percepções, análise acerca da postura dos profissionais de enfermagem durante a realização dos encontros, os movimentos e intervenções realizadas.

O segundo passo foi construído a partir dos acontecimentos identificados no diário que produzem rupturas, que catalisam fluxos, na lógica do processo de intervenção. Denomina-se de analisador este movimento de desnaturalização produzido por estratégias de intervenção de acordo com o modo como os pesquisadores colocam em análise os efeitos das práticas no cotidiano institucional, desconstruindo territórios e sendo capazes de construir novas práticas (Rossi e Passos, 2014).

Um analisador faz, então, no caso deste estudo, o grupo falar, dizer coisas que não são conversadas mesmo estando ali, meio oculta, mas acontecendo.

Esses analisadores foram identificados (o silêncio dos profissionais nas reuniões; o sono; a resistência em sentar na roda; a presença de figuras de poder da instituição durante as reuniões; trocas de saberes a partir das experiências e o desejo de fazer diferente) e, então, trabalhados em forma de esquetes teatrais, apresentados e, posteriormente, levados para a discussão com os participantes.

Os dados coletados pela observação de campo e os discursos produzidos com o grupo, com o uso dos analisadores foram submetidos à análise temática de conteúdo. Para tal, seguiram-se os seguintes passos: transcrição do material resultante da discussão gerada após a apresentação das esquetes; leitura coletiva e; análise do material em grupo de pesquisa. Os participantes receberam nomes fictícios, como forma de preservar o anonimato. 
O grupo de pesquisa constituiu-se em um espaço de produção de conhecimento e análise de dados, onde profissionais e estudantes de outras profissões - grupo multiprofissional - exploraram o material a partir da leitura coletiva. Na sequência, os dados foram depurados e construíram-se as categorias de análise, com a identificação dos analisadores, segundo a AI, que consiste naquilo que expõe elementos do grupo que não eram vistos ou estavam ocultos na dinâmica das relações cotidianas do trabalho, permitindo, assim, a composição dos resultados.

Com base nos dados obtidos e posterior análise, identificaram-se os seguintes núcleos de sentido: Ser ouvido e; A dinâmica processual do trabalho em saúde. O estudo foi submetido ao Comitê de Ética e Pesquisa do Hospital Universitário Antônio Pedro, da Universidade Federal Fluminense (HUAP/UFF), tendo sido aprovado em outubro de 2012 sob n $^{\circ} 125.286$.

\section{RESULTADOS}

Para melhor entendimento dos resultados da pesquisa foram elencados dois grupos de sentidos: Ser ouvido; A dinâmica processual do trabalho em saúde.

\section{Ser Ouvido}

"Ser ouvido" dentro da esfera comunicacional significa ter suas visões, concepções compreendidas e levadas em consideração. Este núcleo de saber está intrinsecamente relacionado com a forma com que o profissional se coloca e participa do processo de trabalho, uma vez que se considera sua voz escutada na organização.

Nesse sentido, nos espaços de discussão, se constatou a coexistência da sensação de os profissionais de enfermagem não serem escutados pelos outros membros da equipe, a dificuldade de empoderamento e a insegurança na maneira de se expressarem.

"Não há nossa voz no hospital, a gente fala e o médico, psicólogo, ninguém escuta." (Dama da Noite)

"É, mas na mesma hora vão concordar, e depois vão descartar isso, o que falamos... Lógico, dificilmente eles vão ouvir e aceitar o que a gente fala e o que a gente acha." (Gardênia)

"Eu acho que também tem um pouco a ver com a timidez, de muitas pessoas não conseguirem expor, que não vem daqui, vem da infância mesmo, que não tem o hábito de estar falando pra outras pessoas." (Ires) Percebe-se que o caso analisador proporcionou o extravasamento dos sentimentos e percepções dos profissionais de enfermagem emergidos no cotidiano do trabalho e, sobretudo, no espaço coletivo.
"Acho que a provocação partiu dessas coisas que foram feitas [...] vem a partir daí, e acho que isso pode ser muito interessante. Puxou a corda, né? Puxou a linha da galera." (Estrelícia)

A EPS possui a potência de promover transformações nas relações, nos processos de trabalho, e isso pode ser constatado nos grupos de discussão.

"Eu até falei que não ia mais a reunião nenhuma, aí eu pensei, 'eu vou na reunião aporrinhar mesmo, eu vou passar a ir à reunião'... É aquele negócio, até eu passar a ser ouvida, entendeu?" (Orquídea)

"Lógico que você tem que se impor, mostrar sua posição. Eu sou o que sou, é claro, se tiver que resolver junto a gente resolve junto, se tiver que passar a frente e pedir ajuda, é claro que você tem que se posicionar. Nós somos necessários mesmo... e me coloco numa posição assim: eu sou técnico de enfermagem com muito orgulho da minha assinatura." (Estrelícia) Percebe-se uma dificuldade por parte dos profissionais de enfermagem se enxergarem incluídos no processo de transformação das práticas em saúde mental, fato constatado pelo silêncio de alguns e pelo sentimento de não 'ser escutado', por outros.

Esse espaço também é identificado como provocador de inquietação, de mudança nas atitudes, além de perceberem o espaço como algo necessário. Há uma sensação de pertencimento e de ideia de grupo.

"Acho que nós mesmos estamos diferentes uns com os outros, estamos melhores no convívio um com o outro." (Gardênia)

Em busca de potencializar o presente espaço de EPS foi proposto o formato de roda para organização daquele ambiente, pois se entende a roda como um dispositivo de articulação a fim de impulsionar transformações nas práticas de cuidado da equipe. Como afirmam Ceccim e Feuerwerer (2004) ao reforçarem que as rodas de EP não são meramente arranjos gerenciais, pois trabalham com processos pedagógicos de organização da rede de atenção à saúde, de qualificação das práticas por meio da integralidade da atenção e da produção por meio de aprendizagens significativas.

"Uma coisa que surgiu como importante foi a organização da roda, em círculo, né?! E antigamente a gente se preocupava muito com isso, fazia um formato de escolinha, e depois a Chuva de Prata propôs que a gente fizesse em círculo." (Copo de leite)

"Só essa coisa, assim, de estar em roda já é uma coisa diferente." (Ires)

"Eu me sinto, assim, até à vontade, se eu quiser perguntar, eu pergunto." (Tulipa) 
"E o interessante é a troca de experiência. Isso aqui é um grupo, você vai conhecendo melhor as pessoas." (Gardênia)

Sentar na roda, fazer parte dela, representa um compartilhamento de responsabilidade; ao "fazer parte da roda" estariam sendo convocados a pensar em soluções para os problemas reais trazidos por eles próprios (para os quais não tinham respostas prontas e alguém externo ao cotidiano deles para dizer o que deveria ser feito) e, diante disso, se transformariam em corresponsáveis pelas decisões tomadas. E, de certo modo, saindo da posição de passivos, indo à caminho da autogestão e saindo do conforto e falso acolhimento oferecido pela heterogestão, que Lourau (2004) define como "geridos" por "outrem", o que significa aceitar o instituído como natural.

As rodas de EP não são meramente arranjos gerenciais, pois trabalham com processos pedagógicos de organização da rede de atenção à saúde, de qualificação das práticas por meio da integralidade da atenção e da produção através de aprendizagens significativas e novas práticas, que se contrapõem à verticalidade do sistema ainda vigente (Castro e Campos, 2014).

\section{A Dinâmica Processual do Trabalho em Saúde}

Este núcleo agregou diferentes condicionantes do processo de trabalho, como jornada extensa, baixa remuneração e envolvimento com os limites humanos, dor e sofrimento psíquico.

"Um dos pontos que eu acho fundamental é que nossa vida, nosso cotidiano fica acorrentado, sai de um pra outro, pra você parar com o colega e perguntar de $\mathrm{A}$ à $\mathrm{Z}$ todos os problemas. Eu acho que a gente não tem tempo pra isso, a gente tem um outro plantão pra pegar, um outro lugar." (Estrelícia)

"E quando está agitado, o telefone tocando o tempo todo, não tem como!” (Gardênia)

"O ti ti ti, cada um pega o seu ônibus e um abraço. Acabou o nosso plantão." (Estrelícia)

"Mas, assim, é difícil, e é isso. Às vezes é difícil até conseguirmos sair para o nosso almoço." (Lavanda do mar)

Pode-se observar através do discurso dos profissionais, que o tempo corrido e a forte rotina de trabalho mostram-se como influenciadores da disposição deles nesse espaço. Assim, muitos encontram nesse espaço um local para descansar e acabam por se sentirem sonolentos.

"Acaba sendo um lugar de descanso. Você passa o dia todo em pé na correria, chega num lugar sentado com ar condicionado, fresquinho." (Ires)
"Depois do almoço, né? Também tem isso, ainda é depois do almoço." (Margarida)

Outro ponto que emergiu a partir das falas dos sujeitos foi o tema abordado no grupo e algumas transformações naquele espaço como fatores que influenciam o interesse e o jeito com que se posicionam.

"Mas têm temas também que convocam mais, né? Tem tema aqui que está todo mundo querendo dar opinião e nem consegue, começa a falar ao mesmo tempo." (Flor do campo)

"Quando você vem com um paciente diferente e ninguém conhece, você se interessa. Como que foi antes? Como que está agora? O que você vai fazer? Eu acho que também tem isso, por exemplo, se você for falar de certos pacientes aquilo já é maçante, o conhecido não é bom, o desconhecido que é bom! É o que você vai poder melhorar." (Tango)

"Bem melhor, muito mais produtivo." (Rosa)

Apesar das dificuldades apontadas por eles, constatase o interesse em discutir e estudar casos ou temas considerados complexos e novos. É como se a necessidade de trocas fosse mais pertinente, mediante um novo cenário, um novo paciente.

Isso aponta para a existência de profissionais sedentos por novos conhecimentos, por vezes, representado pelo o silêncio (sede de ouvir), ou pelo atropelamento das falas (inquietude na busca de apreensão do novo). Seja lá para que lado a balança decline, trará sempre um certo dinamismo ao grupo, um certo desequilíbrio a esse espaço.

Acerca do processo do trabalho em saúde, outro fator se mostrou presente no grupo de discussão, como algo que dificulta a participação de um maior número de profissionais da enfermagem nesse espaço: a dificuldade dos demais membros da equipe multiprofissional em se ocupar do posto de enfermagem para a viabilização do grupo.

\section{DISCUSSÃO}

A trajetória da enfermagem foi marcada por um silêncio diante do predomínio do modelo biomédico a partir da década de 1950, em que a figura do médico era central e à enfermagem cabia cuidar da segurança do paciente, ter a certeza de que o usuário ingeriu a medicação, observar reações, bem como os cuidados de manutenção da vida (Rocha, 2012). Os trabalhadores de enfermagem, então, figuravam como atores coadjuvantes nesse processo, na categoria de executores da ordem disciplinar. 
O forte resquício do passado também pode influenciar na sensação de desvalorização de seu saber, da sua palavra, resultando num posicionamento mais passivo. Há no conflito desses profissionais de enfermagem o motor de sua história e de sua prática, uma profissão que nasceu sob a égide do feminino (Rocha, 2012). No discurso produzido acerca de sua prática, os profissionais de enfermagem parecem afirmar uma cultura profissional, na qual dois dilemas encontramse articulados: um imaginário mimético com relação à medicina e um imaginário feminino de submissão e inferioridade. Entretanto, a potência de equipe está justamente no crescimento coletivo, embora o movimento habitual seja de exclusão. As possibilidades de expressar as diferenças de opiniões, sentimentos, ideias são processos de democratização das instituições, dependente da abertura para acolher as mudanças em si, nos coletivos e nas instituições.

Diante disso, percebe-se que o conceito de empoderamento dialoga com o conceito de EP, na medida em que o mesmo implica em avanço e superação por parte daquele que se empodera (sujeito ativo do processo), superando a simples doação ou transferência pelo estado de benevolência e realizando, assim, por si mesmo, as mudanças e as ações que os levam a evoluir e se fortalecer. É nessa lógica que caminha a estratégia de EP pela sua proposta de reflexão e análise por parte dos profissionais da saúde do seu próprio processo de trabalho, de forma a compartilhar coletivamente a responsabilidade de se construir soluções concretas para os problemas que emergem no cotidiano das suas práticas.

Para se trabalhar nesse contexto, é imprescindível o trabalho em grupo, pois é somente a partir da sensação de pertencimento, partilhamento de objetivos e estabelecimento de relações positivas que se constroem movimentos sólidos na reinvenção de fazeres. É necessário que os profissionais possam se encontrar uns com outros, com as diferenças e semelhanças percebidas nos mais diversos setores em que trabalham e com as experiências de clínicas múltiplas acumuladas. O hábito de refletirem sua assistência, de gerarem um pensamento crítico, de se distanciarem do senso comum e se sentirem à vontade para pensar e agir, a partir da sua própria experimentação da prática, e da sua análise sobre o seu fazer é algo a ser construído e desenvolvido diariamente nesses espaços. E não ocorrerá sem embaraços, sem atropelamentos, sem divergências, sem as dificuldades de se fazerem compreender, simplesmente porque tais passos fazem parte do caminhar dos que almejam a autonomia da palavra.
No que compete à dinâmica processual do trabalho, é preciso ressaltar que no ambiente hospitalar o trabalhador se depara com excessiva carga de trabalho, contato com o limite, tensão, riscos, plantões em diferentes turnos e longas jornadas de atividade. Esses fatores comprometem a integridade física e mental dos trabalhadores de enfermagem (Rocha, 2012).

É comum os profissionais precisarem realizar rodízios de escalas de turnos noturnos e diurnos, duplas ou triplas jornadas de trabalho, em função da baixa remuneração. Soma-se a isso, o fato de que na saúde mental há um intenso envolvimento com os limites humanos, dor e sofrimento psíquico.

Nota-se aqui a relevância do dinheiro como analisador, uma vez que, o dinheiro é um dos analisadores mais sensíveis da socioanálise (Lourau, 2014). A partir dele, é possível manter os diferentes objetivos de vida. A maior parte dos profissionais estudados está, há anos, na condição de prestador de serviço e não possui direitos trabalhistas, o que gera, principalmente para os que ganham menos, uma sensação de insatisfação.

Tais fatores influenciam o desejo e a disposição dos profissionais em buscar novas maneiras de praticar saúde e, por vezes, inviabilizam a participação deles nos espaços de EP.

Se permitir estar em outros lugares, ocupando e se exercendo de outras funções não é uma tarefa fácil e de acontecimento natural, exige trabalho. E este, por sua vez, só é possível por meio da reflexão, da compreensão da importância de um espaço de EPS para qualquer transformação no sentido de se autorizar e ao mesmo tempo se incluir nos processos do cuidado. Nota-se, pois, que a política institucional de EP encaminha-se para uma realidade mais dotada de integralidade, em que os diferentes atores envolvidos no cuidado constroem coletivamente movimentos de transformação das práticas de saúde, com vistas à melhor qualidade do atendimento prestado aos usuários de saúde mental.

Diante de tais resultados, pode-se perceber que o melhor movimento pedagógico não está no seu conteúdo formal, mas naquilo que carrega de potencial. Nesse sentido, se a discussão sobre o processo de trabalho gera inquietação, interroga a forma como se está trabalhando, coloca em dúvida a capacidade de resposta coletiva da unidade de serviço; ou ainda, impede de o trabalhador continuar a ser o mesmo que era, o impede de deixar tudo apenas como está e tenciona suas implicações com os usuários de suas ações, ela conseguiu realizar a EPS. 
Nessa perspectiva é que se percebe a importância do investimento permanente nesses profissionais, compreendendo o seu papel central para uma prática, em que, por um lado o usuário tenha sua autonomia preservada e incentivada nos pequenos fazeres diário e que essa mesma equipe (de enfermagem) possa ir além da reprodução de prescrições técnicas, se posicionando também como um agente terapêutico; por outro, de serem capazes de se perceberem como agentes produtores do cuidado, corresponsáveis no seu processo de trabalho (Muniz, Tavares, Abrahão e Souza, 2015).

Percebemos que os problemas do cotidiano do trabalho podem ser revertidos em questões educacionais, e podem ser empregados como elementos formativos no interior dos serviços, com a participação dos profissionais de saúde.

\section{CONCLUSÃO}

No atual momento em que se encontra a saúde mental, a equipe de enfermagem tem sido convocada a incorporar novas formas de compreender e promover a assistência, o que direciona para a prática voltada à reflexão do processo de trabalho até então construído e a possibilidade de problematização e transformação do mesmo.

Em nosso estudo, foi possível identificar a estratégia de EP como uma prática de ensino-aprendizagem e como uma política de educação na saúde. Um lugar de troca, intercâmbio, de possível 'estranhamento' de saberes e da 'desacomodação' com as práticas vigentes.

Nota-se com a presente pesquisa que há dificuldades para a equipe de enfermagem se fazer presente nesse espaço, tanto pela própria resistência em se permitir ocupar outros lugares, quanto pelos demais membros da equipe de saúde ao não compreenderem o papel da profissão no cuidado ao usuário em transtornos psíquicos. Entretanto, consideram-se tais dificuldades como algo esperado e inerente a qualquer proposta de inovação. Por outro lado, constatou-se haver 'vida' nesse espaço, marcado por tensões, discussões, reflexões no trabalho.

A organização do espaço no formato da roda, ao mesmo tempo em que foi um instrumento visto como resistência por alguns integrantes do grupo, foi compreendida como uma ferramenta capaz de proporcionar dinamismo e aproximação no grupo. $\mathrm{E}$, desse modo, potencializando esses profissionais a se conhecerem, descobrirem e se identificarem como um grupo.
Considera-se importante ressaltar que a EPS se constitui um processo em contínua evolução e adequações. A importância está no grupo poder ir se construindo, se articulando com a possibilidade de reflexão, de análise e construção de soluções coletivas, sendo, portanto, palpável o amadurecimento dos profissionais nesse processo.

\section{IMPLICAÇÕES PARA A PRÁTICA CLÍNICA}

A implementação de espaços de Educação Permanente nos serviços de saúde, na medida que tenciona as práticas proporciona uma ampliação dos espaços de discussão e possibilita um compromisso dos profissionais com melhores condições de assistência à saúde.

\section{REFERÊNCIAS BIBLIOGRÁFICAS}

Abrahão, A. L., e Merhy, E. E. (2014). Formação em saúde e micropolítica: Sobre conceitos ferramentas na prática de ensinar. Interface - Comunicação, Saúde, Educação, 18(49), 312-324. doi: dx.doi. org/10.1590/1807-57622013.0166

Borba, L. O., Guimarães, A. N., Mazza, V, A., e Maftum, M. A. (2012). Assistência em saúde mental sustentada no modelo psicossocial: Narrativas de familiares e pessoas com transtorno mental. Revista da Escola de Enfermagem da USP, 46(6), 1406-1414. Disponível em www.scielo.br/scielo.php?script=sci_ arttext\&pid=S0080

Castro, C. P., e Campos, G. W. S. (2014). Apoio institucional paidéia como estratégia para educação permanente em saúde. Revista Trabalho Educação e Saúde, 12(1), 29-50. Disponível em www.scielo.br/ scielo.php?script=sci_arttext\&pid=S1981

Ceccim, R. B. (2012). Desenvolvimento de competências no trabalho em saúde: Educação, áreas do conhecimento e profissões no caso da saúde. Tempus: Actas de Saúde Coletiva. 6(2), 253-277. Disponível em http://www.tempus.unb.br/index.php/tempus/ article/view/1128

Lourau, R. (2014). Análise institucional. Petrópolis: Editora Vozes. 
Muniz, M. P., Tavares, C. M., Abrahão, A. L., eSouza, Â. C. (2015). A assistência de enfermagem em tempos de reforma psiquiátrica. Revista Portuguesa de Enfermagem deSaúde Mental(13), 61-65. Disponível em http:// www.scielo.mec.pt/pdf/rpesm/n13/n13a08.pdf

Rocha, R. M. (2012). Enfermagem em saúde mental. Rio de Janeiro: Senac Nacional.

Rossi, A., e Passos, E. (2014). Análise institucional: Revisão conceitual e nuances da pesquisa-intervenção no Brasil. Revista EPOS, 5(1), 156-181. Disponível em http://pepsic.bvsalud.org/pdf/epos/v5n1/09.pdf
Santos, C. G., Portugal, F. T. A., Silva, M. A. B., Souza, Â. C. S., e Abrahão, A. L. (2015). Formação em saúde e produção de vínculo: Uma experiência PET-Saúde na rede de Niterói, RJ, Brasil. Interface - Comunicação, Saúde, Educação, 19(1), 985-993. doi: dx.doi. org/10.1590/1807-57622014.0868

Slomp Junior, H., Feuerwerker, L. C. M., \& Land, M. G. P. (2015). Educação em saúde ou projeto terapêutico compartilhado? O cuidado extravasa a dimensão pedagógica. Ciência e Saúde Coletiva, 20(2), 537-546. doi:dx.doi.org/10.1590/1413-81232015202.00512014

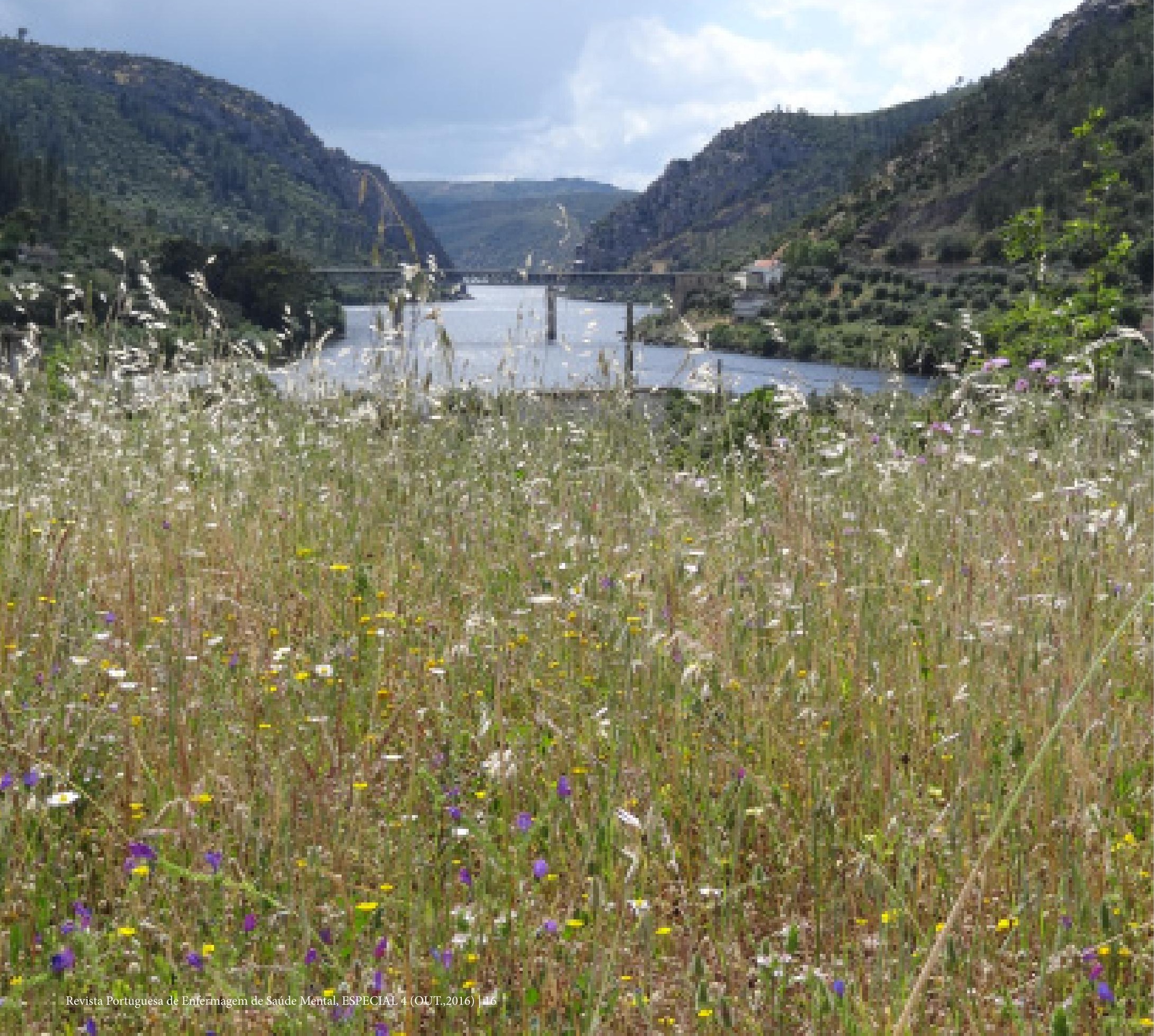

